\section{Neuropsychiatry and behavioural psychology}

Neuropsychiatry and Behavioural Neurology Explained. Alex J Mitchell. Pp. 525. Edinburgh: Saunders. 2004. ISBN 0-70202688-3.

Neuropsychiatry is an exciting and rapidly evolving field of psychiatry. Man has always been fascinated by the body-mind dichotomy and how it is integrated. In this book, Mitchell explores this in a systematic yet exciting manner.

The book is organised in five main sections. In section I, the principles of neuropsychiatric assessment are discussed. Section II covers psychiatric complications of neurological disorders. In section III, delirium and the various dementias are discussed. Section IV provides an overview of treatments in neuropsychiatry and section $\checkmark$ provides a very interesting and useful discussion on the pathogenesis of different neuropsychiatric symptoms and syndromes.

This is followed by an epilogue where everything is brought together in a chapter called 'Maxims of neuropsychiatry revisited'. The appendix includes various useful and interesting features, including a timeline with historical landmarks in neuropsychiatry, useful web addresses, recommended assessment scales and a list of recommended reading.

The book is written in an understandable and easy-to-read style. It is colourful and has lots of useful illustrations and text boxes to supplement the text that help to keep the reader's attention. The content is comprehensive, evidence-based and up to date. Each chapter is usefully summarised at the end.

A possible shortcoming is that references are made to various neuropsychological and neuropsychiatric test batteries which at times are not explained further, leaving the inexperienced reader in the dark. A useful supplement to the text would have been clinically useful, easy-to-do bedside tests for different cognitive functions. The chapters on HIV and neurosyphilis could have been more detailed for the doctor working in the South African context.

Despite this criticism, I found this an outstanding book. It is a very useful text for anyone interested or working in the field of neurology or neuropsychiatry, especially psychiatrists and psychologists. Trainees in psychiatry would find this a valuable and practical resource for clinical work, as well as an indispensable text for exam preparation. I can recommend it to all interested in this growing and important field of psychiatry.

\section{Lize Weich}

